\title{
Evaluation of Piezodiagnostics Approach for Leaks Detection in a Pipe Loop
}

\author{
Jhonatan Camacho-Navarro", a , Magda Ruiz ${ }^{1, ~ b}$, Rodolfo Villamizar ${ }^{2, ~ c, ~}$ \\ Luis Mujica ${ }^{1, d}$, Oscar Pérez ${ }^{2, e}$ \\ ${ }^{1}$ Department of Mathematics, Escola d'Enginyeria de Barcelona Est (EEBest), \\ Campus Diagonal-Besós, Universitat Politécnica de Catalunya (UPC) BARCELONATECH, \\ Comte d'Urgell, 187, E-08036, Barcelona, Spain \\ ${ }^{2}$ Escuela de Ingenierías Eléctrica, Electrónica y de Telecomunicaciones (E3T), Universidad \\ Industrial de Santander (UIS). Grupo de Control Electrónica Modelado y Simulación (CEMOS). \\ Santander, Colombia \\ acamacho.navarro.jhonatan@gmail.com, ${ }^{\mathrm{b}}$ magda.ruiz@upc.edu, ${ }^{\mathrm{c}}$ rovillam@uis.edu.co, \\ duis.eduardo.mujica@upc.edu, eoscar.perez.gamboa@gmail.com
}

Keywords: Piezodiagnostics, Pipe Leak detection, Principal Component Analysis, Self-Organizing Maps, Environmental and operation conditions.

\begin{abstract}
Pipe leaks detection has a great economic, environmental and safety impact. Although several methods have been developed to solve the leak detection problem, some drawbacks such as continuous monitoring and robustness should be addressed yet. Thus, this paper presents the main results of using a leaks detection and classification methodology, which takes advantage of piezodiagnostics principle. It consists of: i) transmitting/sensing guided waves along the pipe surface by means of piezoelectric device ii) representing statistically the cross-correlated piezoelectric measurements by using Principal Component Analysis iii) identifying leaks by using error indexes computed from a statistical baseline model and iv) verifying the performance of the methodology by using a Self Organizing Map as visualization tool and considering different leak scenario. In this sense, the methodology was experimentally evaluated in a carbon-steel pipe loop under different leaks scenarios, with several sizes and locations. In addition, the sensitivity of the methodology to temperature, humidity and pressure variations was experimentally validated. Therefore, the effectiveness of the methodology to detect and classify pipe leaks, under varying environmental and operational conditions, was demonstrated. As a result, the combination of piezodiagnostics approach, cross-correlation analysis, principal component analysis, and Self Organizing Maps, become as promising solution in the field of structural health monitoring and specifically to achieve robust solution for pipe leak detection.
\end{abstract}

\section{Introduction}

Leaks are the main source of incidents and damage types reported by fluid transportation companies, which cause abnormal operation and possible adverse consequences. For instance, the US Department of Transportation Pipeline and Hazardous Materials Safety Administration (PHSA) has reported around 11.192 incidents in the last 20 years period, which has generated costs by approximately MMUS\$ 6.800, corresponding to hazardous liquid incidents [1]. Bashtransgaz Ltd has documented another accidents, where people were affected due to ignition of natural gas transported by Urengoy-Petrovsk GP.

Since these accidents affect the integrity of surrounding structures (i.e. buildings, farms, etc), human safety and have great economical impacts, it is necessary to obtain a leak detection system with high reliability, in order to reduce maintenance costs and minimize accidents occurrence. In this sense, structural health monitoring (SHM) methodologies can be implemented in order to achieve robustness, precision and sensitivity characteristics specified on international standards as API 1155. 
According to above requirements, in this paper a methodology for SHM based on piezodiagnostics and principal component analysis was used to detect and classify pipe leaks by means of self-organizing maps. Experimental data from a carbon-steel pipe loop was used to validate the effectiveness of the methodology, and its robustness considering environmental and operational variations.

\section{Pipe leak damage assessment methodology}

Pipe leaks detection and classification are achieved by implementing three stages (See Fig 1.): $i$.) Data acquisition, ii.) Statistical feature extraction and iii.) Clustering.

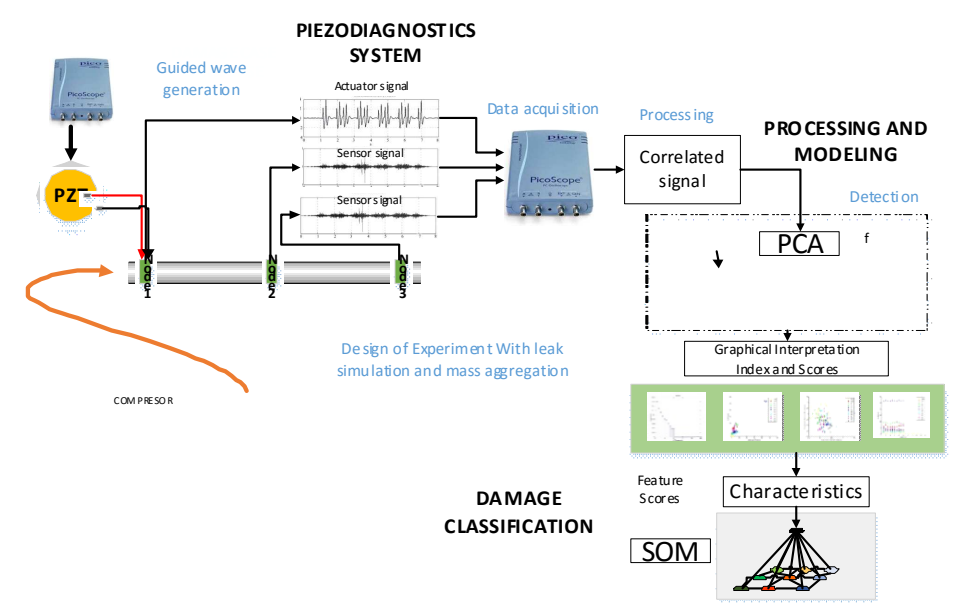

Figure 1. Components of the pipe leak detection and classification methdoology

In the data acquisition stage is used the piezo-diagnostics principle, by bonding piezoelectric devices to the surface structure, where one of them operates as actuating element and the other ones as sensors. Several works have demonstrated the high sensitivity of guided waves generated by PZT devices to scattering, reflection, and mode conversion due to structural discontinuities, which makes possible to identify structural damages [2, 3]. After piezoelectric records are collected, crosscorrelation is computed between actuating and sensing signals with the objective of reducing common noise and minimizing data trends effects.

The statistical feature extraction stage comprises two procedures: data reduction through principal component analysis (PCA) and statistical indexes computation. By means of PCA a minimal representation of cross-correlated signals is obtained to represent the data variance in a reduced space with minimal redundancy. If records from undamaged state of the structure are unfolded in the $2 \mathrm{D}$ matrix $\mathrm{X}$, then a baseline statistical model corresponding to pristine measurements can be expressed through PCA by means of Eq. $1[4,5]$.

$$
\mathrm{X}=\mathrm{TP}^{\mathrm{T}}+\mathrm{E}=\text { model }+ \text { noise }
$$

Where, $\mathrm{P}$ is a linear transformation matrix that relates the data matrix $\mathrm{X}$ in the new coordinates and it is known as the principal components. $\mathrm{T}$ is the projected matrix to the reduced space and the noise E-matrix describe the residual variance neglected by the statistical model. Once the PCA model is built, $Q$ and $T^{2}$ statistical indexes (defined by Eq. (2)) are used to determine the existence or absence of pipe leaks [6], where differences between statistical indexes regarding to baseline and current state are attributed to leak presence. The Q-statistic is defined as a lack of fit measure between the analyzed experiment and the baseline records. Likewise, the Hotelling $\mathrm{T}^{2}$ statistic indicates how far each trial is from the center $(\mathrm{T}=0)$ of the reduced space of coordinates.

$$
Q=\sum_{j}\left(e_{j}\right)^{2} \quad \text { and } \quad T^{2}=T^{\prime} \lambda^{-1} T
$$


Where, $e_{j}$ is the residual error for each $j-t h$ principal component used to reconstruct the trial experiment and $\lambda$ (singular values) are the respective variances of the reduced-space.

As a final stage, a Self-Organizing Map (SOM) is used for pipe leaks classification, which has capabilities to cluster cases in similar damage types. The SOM network maps input features (statistical indexes) into neighbor clusters by means of competitive learning facilitating pipe leaks recognition [6].

\section{Pipeline case study}

Experimental data from a carbon-steel pipe loop with five $100 \times 2.54 \times 0.3 \mathrm{~cm}$ (length, diameter, thickness) sections was used to validate the effectiveness of the methodology for leaks detection and classification. The piezoactive system consists of an AWG generator, data acquisition hardware, noise filters, amplifiers and three PZT devices per section, where the PZT bonded in the middle point of each section operates as actuating element and the remaining ones as sensors. Operational conditions are controlled through bridles, a valve, a manometer and a compressor, whilst environmental conditions are varied by means of high power lamps and a trough-shaped vessel filled with water. Thus, humidity changes are produced by regulating the water state (moist air/steam flow) in the vessel by means of coolers and heating resistors, while temperature variations are controlled by adjusting the electrical power in the lamps.

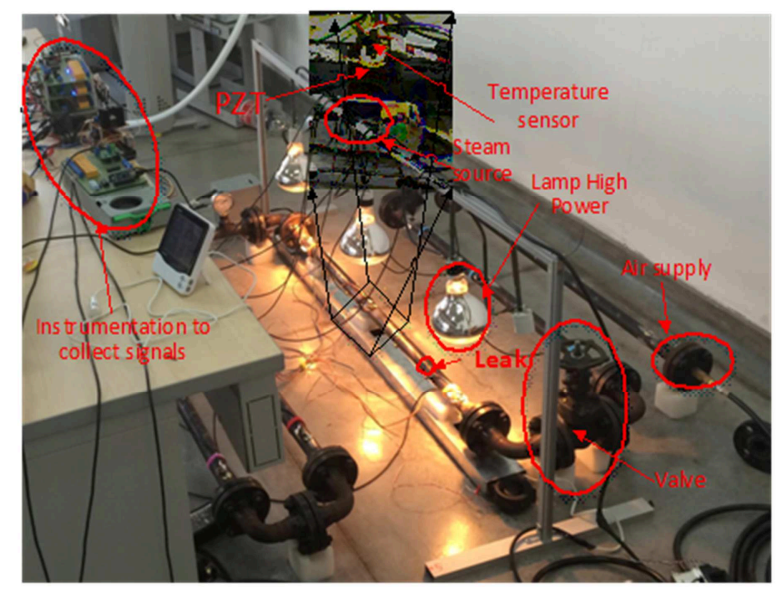

Figure 2. Experimental setup

A leak was induced by a full opening of a hole between the PZT devices (Actuator-Sensor) in the third section of the pipe loop structure in order to recreate damage condition. Also, an external object was added in order to evaluate the capability to distinguish between damage types.

\section{Results and discussion}

The experimental scenario was configured at 80 PSI and recording 100 experimental repetitions regarding to undamaged and the respective damage states. In addition, temperature/humidity conditions were varied in range of [26-40] ${ }^{\circ} \mathrm{C}$ and [40-80] \% RH respectively. The guided waves were generated by exciting the PZT actuators around its resonance frequency $(\sim 100 \mathrm{KHz})$ and then these signals were amplified to $\pm 10 \mathrm{~V}$.

Figure 3a presents the statistical indexes for a leak induced in the pipe loop, where $\mathrm{T}_{0}, \ldots, \mathrm{T}_{11}$ are different temperature/humidity conditions collected each 10 minutes. According to Figure 3a, clusters for each environmental condition are observed and it is possible to distinguish between nominal (undamaged) and leak states. 

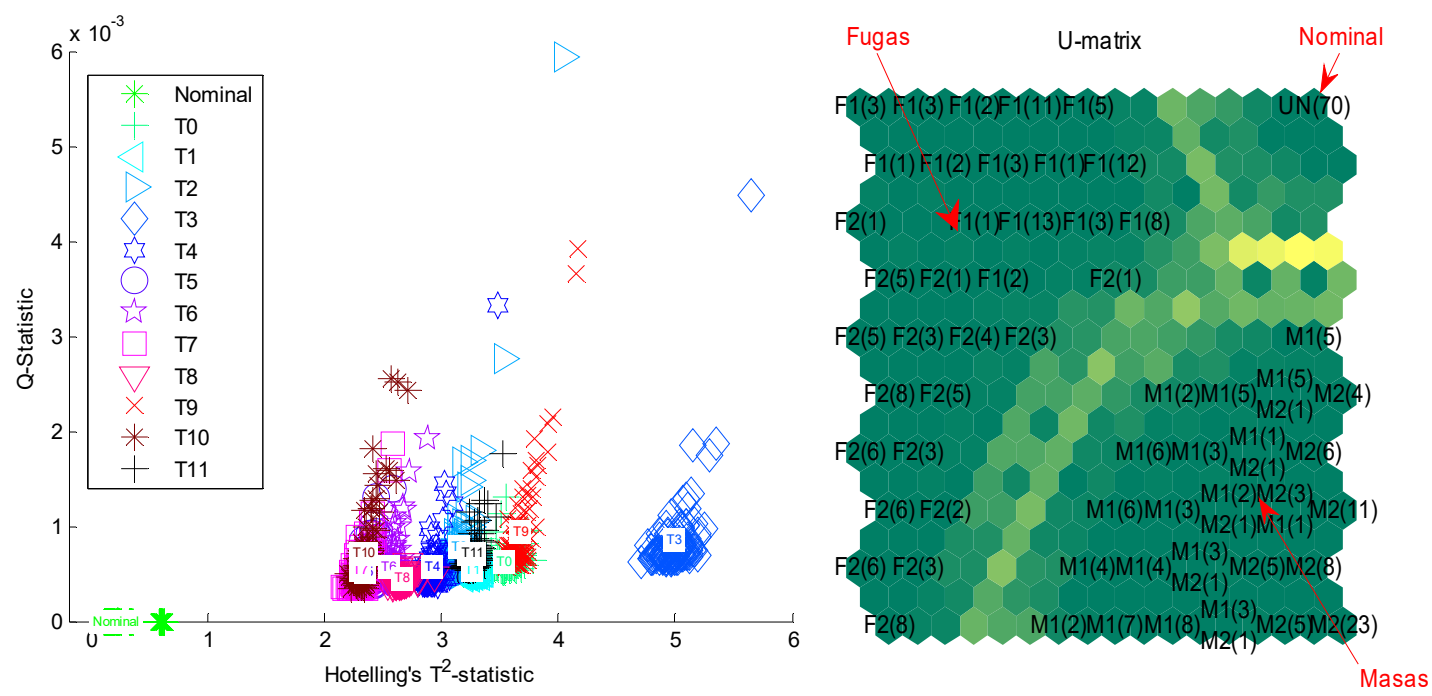

Figure 3. a.) Leak at different environmental conditions. b.) Detection of leak and mass adding damage types.

Figure $3 \mathrm{~b}$ presents the clusters obtained with a SOM map for leak and mass adding damage types. The analyzed data corresponds to experimental records collected without varying environmental conditions. It is noted clear boundaries between damage types, which is a good characteristic of classification systems.

\section{Conclusion}

The methodology for leak detection under varying environmental conditions presented in this paper, experimentally shown its feasability to detect leaks with a good performance, but also a robustness to temperature/humidity variations was obtained since is it possible to differenciate enviromental changes respect to leaks.

\section{References}

[1] Information on https://hip.phmsa.dot.gov/analyticsSOAP/saw.dll?Portalpages. Available at April, 2016.

[2] J. S. Crider, Damage detection using lamb waves for structural health monitoring. Department of the air force air university, vol. THESIS, pp. 1-97, 2007.

[3] Mavi, G. S. (2013). Feasibility of using ultrasonic guided waves for under water pipe inspection (Doctoral dissertation) THAPAR UNIVERSITY PATIALA).

[4] Tibaduiza, D. A., Mujica, L. E., \& Rodellar, J. (2011). Comparison of several methods for damage localization using indices and contributions based on PCA. In Journal of Physics: Conference Series (Vol. 305, No. 1, p. 012013). IOP Publishing.

[5] Mujica, L. E., Rodellar, J., Fernandez, A., \& Guemes, A. (2010). Q-statistic and T2-statistic PCA-based measures for damage assessment in structures.Structural Health Monitoring, 1475921710388972.

[6] Hyötyniemi, H. (2001). Multivariate regression: Techniques and tools. Helsinki University of Technology. 